\title{
Developing Rapid and Advanced Visualisation of Magnetic Structures Using 2-D Pixelated STEM Detectors
}

\author{
Magnus Nord ${ }^{1}$, Matus Krajnak ${ }^{1}$, Rantej Bali ${ }^{2}$, Gregor Hlawacek ${ }^{2}$, Vico Liersch ${ }^{2}$, Jürgen Fassbender $^{2}$, \\ Stephen McVitie ${ }^{1}$, Gary W. Paterson ${ }^{1}$, Ian Maclaren ${ }^{1}$, Damien McGrouther ${ }^{1}$ \\ 1. School of Physics and Astronomy, University of Glasgow, Glasgow, United Kingdom \\ 2. Institute of Ion Beam Physics and Materials Research, Helmholtz-Zentrum Dresden-Rossendorf, \\ Dresden, Germany
}

Transmission Electron Microscopy (TEM) electron diffraction patterns, imaged from the back focal plane of the objective lens, reveals rich information about the structure of materials. The sharpest patterns are obtained using a parallel (semi-convergence angle $<1 \mathrm{mrad}$ ) electron beam which typically illuminates a circular region with a diameter of $100 \mathrm{~nm}$. In Scanning Transmission Electron Microscopy (STEM) the electron beam is focused to form a fine probe, potentially with sub-Ångström diameter. Signals generated by the interaction of the probe with a sample are collected by detectors which integrate the scattered electron intensity in the back focal plane for each probe position in a 2-D scan. A key aspect to obtaining high resolution information is that the performance of scanning and detection should be performed rapidly in order to provide live imaging for the user and to also to mitigate the effect of microscope instabilities and specimen drifts.

Recently, advances in fast direct electron counting systems have enabled the development of 2-D pixelated detectors with high acquisition speeds. Applying these to the STEM technique enables imaging of the contents of the back focal plane at high frame rates, i.e. the full diffraction pattern can be acquired for every scan position in a STEM dataset. This yields a 4-D dataset consisting of two spatial probe position dimensions, and two reciprocal detector positions. While this opens up many new and exciting research avenues, there are still many challenges in how to use the 4-D datasets optimally. In this work we focus on solutions for performing live or near-live magnetic imaging using a fast pixelated detector in a scanning transmission electron microscope.

The experimental work was performed on a probe corrected Jeol ARM200cF equipped with a Medipix3 fast pixelated detector capable of acquiring 1100 frames per second. To image magnetic induction in the materials the STEM was operated in Lorentz mode [1], which involves turning off the objective lenses such that the sample resides in a near-field-free environment. A custom aberration corrector mode was utilised to enable probe semi-convergence angles from 0.4-3.0 mrad with corresponding probe diameters from $<1 \mathrm{~nm}$ to $6 \mathrm{~nm}$ [1]. For magnetic samples, the Lorentz interaction with the beam results in electron wave phase changes (equivalent to beam deflections in the classical perspective) proportional to the magnetic induced phase gradients across the sample. Using a pixelated detector, the direction and strength of the integrated magnetic induction of the sample can be quantitatively measured by tracking the angular deflection of the bright field disk.

This presentation will focus on solutions for live imaging of magnetic structures in FeAl thin films patterned with $\mathrm{Ne}^{+}$ions to form a ferromagnetic disc within the paramagnetic film, as shown in Fig. 1. An example of magnetic contrast is shown in (c-h), where the contrast within the disc is due to Lorentz deflection of the electron beam. The figures show two different methods for extracting this deflection, 
with vastly different degrees of computational cost. The images in (c-f) are formed using different single pixels at the edge of the bright field disk indicated by the respective labels in (b). In (g) and (h) the more advanced edge detection technique was used to determine the position of the disk in two orthogonal directions. As seen in Fig. 1, the edge detection technique clearly produces increased levels of magnetic contrast and signal-to-noise ratio, but it is much more computationally demanding. Thus, the more simple method is more suited for live imaging. We will also show how offloading the data processing to central and graphical processing units allows for rapid data processing with different methods. This includes the single pixel and edge detection methods explained above, as well as centre of mass methods. Lastly, the processed data is transferred to an image processing software such as Digital Micrograph [2] using open and standard network components, which enables control of the detector system, including choice of analysis technique, and the resulting images to be viewed in real-time on a single machine. The presentation will also touch on file formats chosen for data storage and ways of visualising the 4-D datasets [3].

[1] S. McVitie et al, Ultramicroscopy 152 (2015), p. 57.

[2] By Gatan Inc.

[3] The authors are indebted to the EPSRC for the funding of this work via the project "Fast Pixel Detectors: a paradigm shift in STEM imaging" (Grant reference EP/M009963/1).
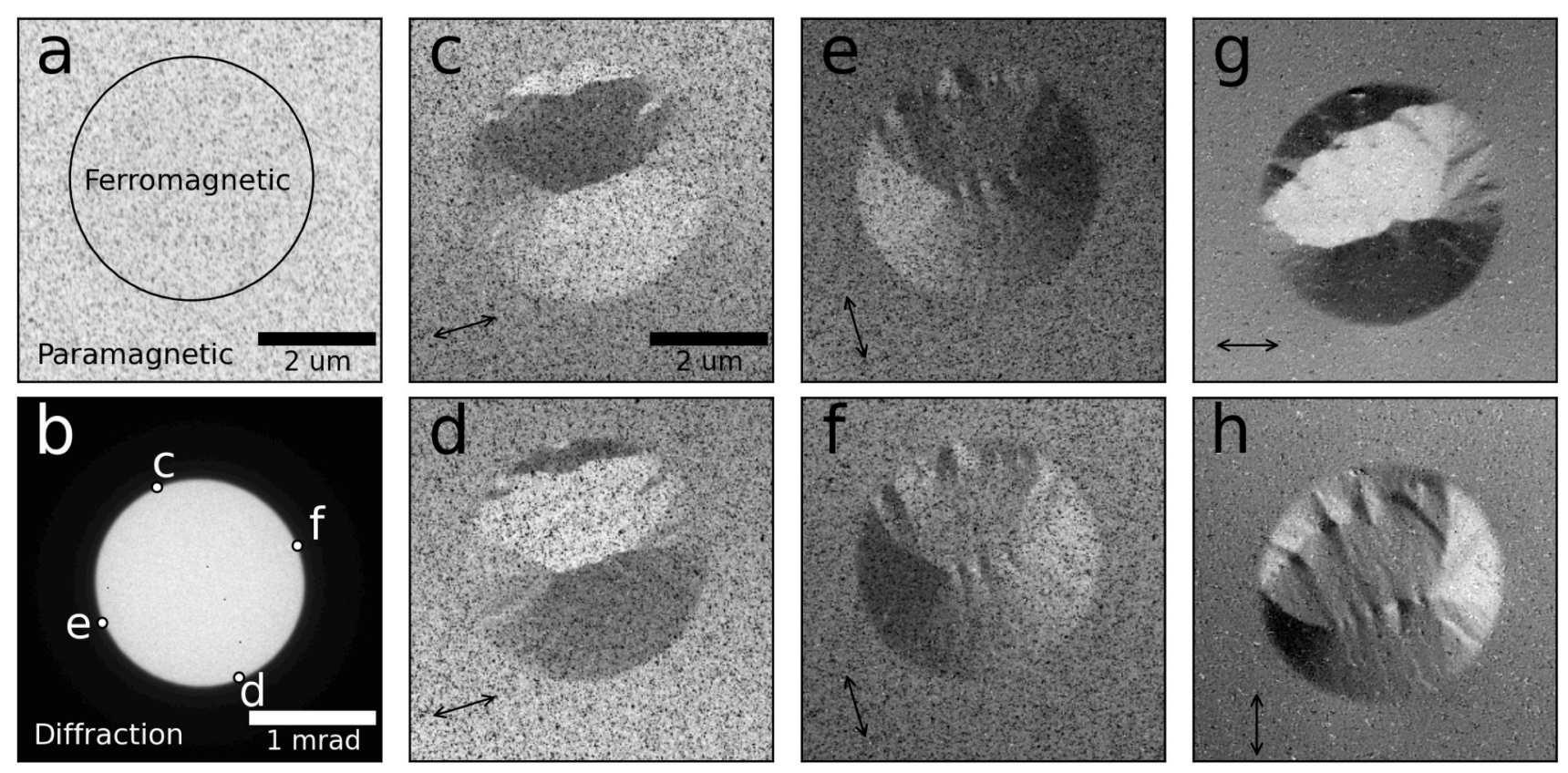

Figure 1. STEM images of $\mathrm{Ne}^{+}$ion patterned FeAl from a single 4-D dataset. (a) Bright Field image. Region within the circle has been patterned. (b) Sum over all the probe positions, giving an average bright field disk. Showing the location of the single pixel synthetic aperture used in (c-f). (c-f) Magnetic contrast from shifts in the bright field disk, using a single pixel in (b). (g-h) Magnetic contrast using edge detection on the bright field disk. (g) Horizontal and $(\mathrm{h})$ vertical magnetic induction. The double headed arrows indicate the component of integrated magnetic induction mapped. 